\title{
Differential Tissue-specific and Pathway-specific Anti-obesity Effects of Green Tea and Taeumjowitang, a Traditional Korean Medicine, in Mice
}

\author{
Junil Kim ${ }^{1,2}$, Sujin Park ${ }^{3}$, Haein $\mathrm{An}^{3,4}$, Ji-Young Choi ${ }^{5}$, Myung-Sook Choi ${ }^{5}$, Sang-Woon Choi ${ }^{6}$, Seong-Jin Kim ${ }^{3,7}$ \\ ${ }^{1}$ The Institute for Diabetes, Obesity, and Metabolism and ${ }^{2}$ Deparment of Genetics, Perelman School of Medicine at the University of Pennsylvania, \\ Philadelphia, PA, ${ }^{3}$ Precision Medicine Research Center and Advanced Institutes of Convergence Technology, Seoul National University, ${ }^{4}$ Department \\ of Biological Sciences, Sungkyunkwan University, Suwon, ${ }^{5}$ Center for Food and Nutritional Genomics Research and Department of Food Science \\ and Nutrition, Kyungpook National University, Daegu, ${ }^{6}$ Chaum Life Center, CHA University School of Medicine, Seoul, ${ }^{7}$ Department of \\ Transdisciplinary Studies Graduate School of Convergence Science and Technology, Seoul National University, Suwon, Korea
}

\begin{abstract}
Background: Traditional medicines have been leveraged for the treatment and prevention of obesity, one of the fastest growing diseases in the world. However, the exact mechanisms underlying the effects of traditional medicine on obesity are not yet fully understood. Methods: We produced the transcriptomes of epididymal white adipose tissue (eWAT), liver, muscle, and hypothalamus harvested from mice fed a normal diet, high-fat-diet alone, high-fat-diet together with green tea, or a high-fat-diet together with Taeumjowitang, a traditional Korean medicine.

Results: We found tissue-specific gene expression patterns as follows: (i) the eWAT transcriptome was more significantly altered by Taeumjowitang than by green tea, (ii) the liver transcriptome was similarly altered by Taeumjowitang and green tea, and (iii) both the muscle and hypothalamus transcriptomes were more significantly altered by green tea than Taeumjowitang. We then applied integrated network analyses, which revealed that functional networks associated with lymphocyte activation were more effectively regulated by Taeumjowitang than by green tea in the eWAT. In contrast, green tea was a more effective regulator of functional networks associated with glucose metabolic processes in the eWAT.
\end{abstract}

Conclusions: Taeumjowitang and green tea have a differential tissue-specific and pathway-specific therapeutic effect on obesity.

(J Cancer Prev 2017;22:147-158)

Key Words: RNA sequencing, Traditional medicine, Obesity, Gene regulatory networks

\section{INTRODUCTION}

Obesity is one of the fastest growing health conditions in the world and comes with a significant risk for numerous health problems. It is associated with the development of metabolic syndrome, resulting in dyslipidemia, insulin resistance, glucose intolerance, and chronic inflammation. ${ }^{1}$ This chronic inflammation often leads to hepatic steatosis, type 2 diabetes mellitus, cardiovascular disease, hypercholesterolemia, hyperglyceridemia, nonalcoholic fatty liver disease, arthritis, asthma, and even some types of cancer. ${ }^{2-4}$

Due to the complexity of obesity, traditional oriental medicine, which uses natural products that typically are composed of multiple interacting components and can have multiple targets, ${ }^{5,6}$ has emerged as a new alternative medicine for the treatment of obesity. For example, the positive anti-obesity effects of green tea are well known, and are due to the synergistic interactions of the multiple components in green tea. ${ }^{7,8}$ It has been suggested that green tea increases thermogenesis and fat oxidation due to its high content of catechin polyphenols and caffeine. ${ }^{9}$ Moreover,

Received August 5, 2017, Revised August 31, 2017, Accepted August 31, 2017

Correspondence to: Seong-Jin Kim

Precision Medicine Research Center and Advanced Institutes of Convergence Technology, Seoul National University, 145 Gwanggyo-ro, Yeongtong-gu, Suwon 16229, Korea

Tel: +82-31-888-9982, Fax: +82-31-888-9983, E-mail: jasonsjkim@snu.ac.kr, ORCID: Seong-Jin Kim, http://orcid.org/0000-0003-4335-8793

Copyright (C) 2017 Korean Society of Cancer Prevention

(c) This is an Open Access article distributed under the terms of the Creative Commons Attribution Non-Commercial License (http://creativecommons.org/licenses/by-nc/4.0) which permits unrestricted non-commercial use, distribution, and reproduction in any medium, provided the original work is properly cited. 
green tea has also been shown to attenuate obesity-related physiological conditions, such as insulin resistance, ${ }^{10}$ hypercholesterolemia, and hyperglycemia. ${ }^{11}$

Taeumjowitang is a traditional Korean medicine composed of eight herbs/plant components (Radix Platycodi, Semen Raphani, Semen Coicis, Semen Castaneae, Fructus Schisandrae, Radix Liriope platyphylla, Rhizoma Acori gramineus, and Herba Ephedrae) that is used as an anti-obesity treatment. ${ }^{12}$ The efficacy and safety of Taeumjowitang has already been assessed in obese Korean children ${ }^{13}$ and adults. ${ }^{14}$ Among the various components of Taeumjowitang, platycodin D, a major component of Radix Platycodi, is the most widely studied for both its anti-obesity effects $^{15-17}$ and its protective effects against conditions associated with obesity, such as cardiovascular conditions, ${ }^{18,19}$ inflammation, ${ }^{20}$ and cancer. ${ }^{21}$ The anti-obesity-related efficacy and safety of Taeumjowitang may result from the multi-component nature and multi-target effects provided by the eight components that comprise this medicine.

Transcriptomic studies are a common approach for the identification of molecular mechanisms underlying the anti-obesity effects of traditional medicine. ${ }^{22-24}$ Traditional medicines with anti-obesity effects have been demonstrated to decrease the expression of genes associated with lipid metabolism. However, these studies have not tried to compare the differential mechanisms of traditional medicines.

In this study, we performed a large-scale transcriptomic analysis of four murine tissues (epididymal white adipose tissue [eWAT], liver, muscle, and hypothalamus) from a diet-induced-obesity animal model and investigated whether green tea and Taeumjowitang have differential tissue- and pathway-specific therapeutic effects on obesity. To the best of our knowledge, this is the first study describing the tissue- and pathway-specific transcriptome modulations of two traditional medicines with anti-obesity effects.

\section{MATERIALS AND METHODS}

\section{Animal models}

Four-week-old male C57BL/6J mice (Jackson Laboratory, Bar Harbor, ME, USA) were randomly assigned to four groups: normal diet ( $17.2 \mathrm{kcal} \%$ dietary fat, $\mathrm{n}=31$ ), high-fat-diet ( $60 \mathrm{kcal} \%$ dietary fat, $\mathrm{n}=17)$, high-fat-diet $+3 \%(\mathrm{w} / \mathrm{w})$ Taeumjowitang $(\mathrm{n}=20)$, and high-fat-diet $+0.25 \%(w / w)$ green tea ethanol extract $(n=19)$ for 12 weeks. The composition rate of eight components in Taeumjowitang added to the diet was informed by a previous study. ${ }^{12}$ The doses of Taeumjowitang and green tea were selected by the criteria of the Korean Ministry of Food and Drug Safety. The human dose of Taeumjowitang is $9 \mathrm{~g} / \mathrm{d}$ for adults and that of green tea is 300 to $500 \mathrm{mg} / \mathrm{d}$. We translated the dosages of Taeumjowitang and green tea from human to mouse using the body surface area normalization method. ${ }^{25}$ All experiments were performed in accordance with protocols for animal studies approved by the Ethics Committee of Kyungpook National University (approval No. KNU 2012-136).

\section{2. mRNA sequencing}

Three mice were randomly selected from each dietary group and eWAT, liver, muscle, and hypothalamus tissue samples were collected from each mouse. Total RNA was extracted from the tissues using TRIzol Reagent (Invitrogen Life Technologies, Grand Island, NY, USA). After synthesizing cDNA libraries, the cDNA library quality was evaluated using an Agilent 2100 BioAnalyzer (Agilent, Santa Clara, CA, USA) and quantified using the KAPA Library Quantification Kit (Kapa Biosystems, Wilmington, MA, USA). After cluster amplification of the denatured templates, samples were sequenced by an Illumina HiSeq 2500 ( $2 \times 150$ bp; Illumina San Diego, CA, USA).

\section{Preprocessing the mRNA-seq data}

We filtered the sequences, discarding low quality reads from the dataset according to the following parameters: reads comprised of $>10 \%$ ambiguous $(\mathrm{N})$ bases, reads with $>40 \%$ of bases with a phred quality score $<20$, and reads with an average phred quality score $<20$. The resulting high quality reads were mapped onto the human reference genome (Ensembl release 72) using the aligner software STAR, v.2.3.0e. ${ }^{26}$ Gene expression levels were measured using Cufflinks v2.1.1, ${ }^{27}$ the gene annotation database included in Ensembl release 72. Non-coding gene regions were removed by employing the mask option. To improve accuracy, the 'multiread correction' and 'frag bias-correct' options were used. All other parameters were set to default values.

\section{Differentially expressed gene and gene ontology analyses}

We identified differentially expressed genes (DEGs) in the following comparison groups: HD vs. ND, TH vs. HD, and GH vs. HD. Gene expression levels were generated using HTSeq-count v0.5.4p3, ${ }^{28}$ and DEGs were identified for each comparison group using the R package TCC. ${ }^{29} \mathrm{TCC}$ uses robust normalization strategies to compare tag count data; normalization factors were calculated using the iterative DEGES/edgeR method. Genes were considered DEGs if their fold-change between comparison groups 
was at least 2-fold and with a $P$-value $<0.05$. We analyzed gene ontology (GO) enrichment using the DAVID functional annotation tools. $^{30}$

\section{Construction of the integrated epididymal white adipose tissue gene regulatory network}

To identify significant regulators of the eWAT transcriptome response to the various diets in this study, we constructed an integrated eWAT gene regulatory network (GRN). First, we retrieved a reliable human green regulatory network from two studies; the study by Gerstein et al. ${ }^{31}$ and SignaLink version $2 .^{32}$ We then compiled lists of genes to fit into the six DEG groups: UHDTG (up-regulated by HD and down-regulated by Taeumjowitang and green tea), UHDT (up-regulated by HD and down-regulated by Taeumjowitang), UHDG (up-regulated by HD and down-regulated by green tea), DHUTG (down-regulated by HD and up-regulated by Taeumjowitang and green tea), DHUT
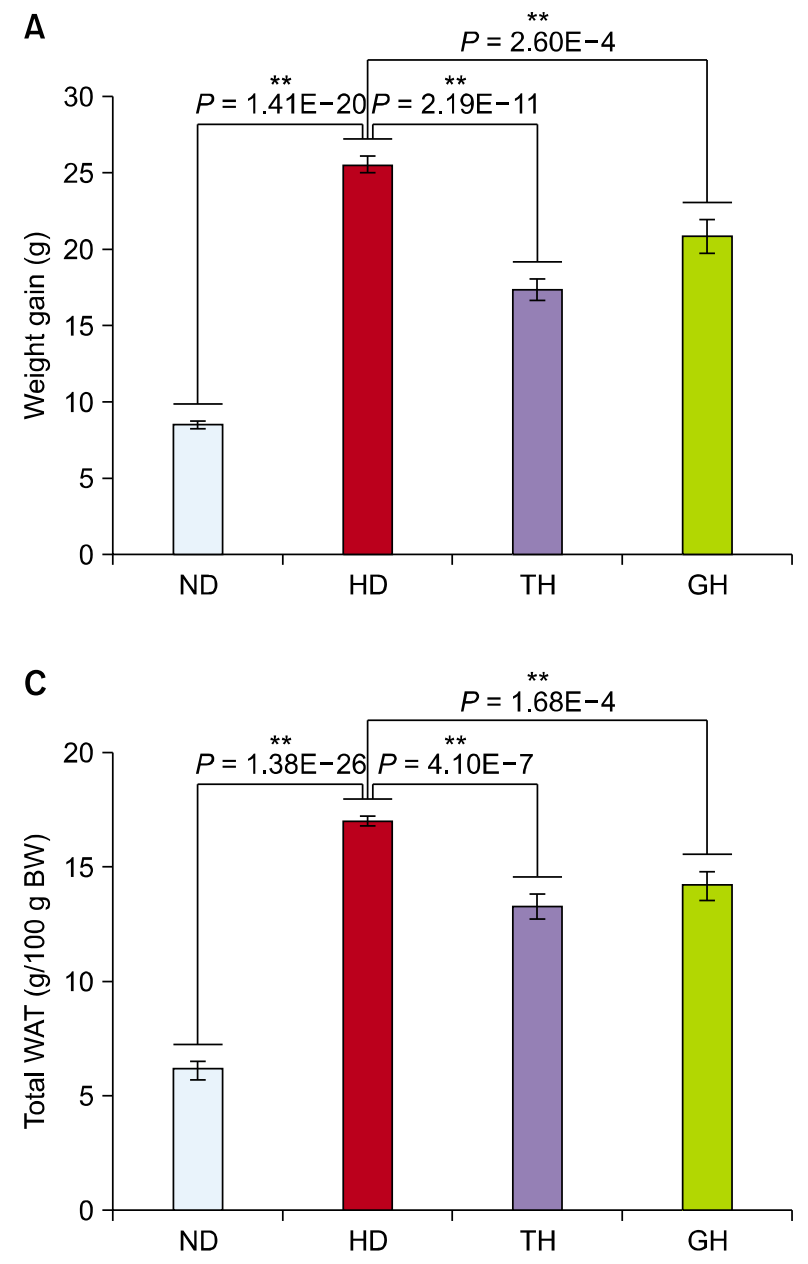

(down-regulated by HD and up-regulated by Taeumjowitang), and DHUG (down-regulated by HD and up-regulated by green tea). Statistically significant transcription factors (TFs) for each DEG group were identified using hypergeometric tests to compare DEG groups with target gene groups by two criteria: a $P$-value $<$ 0.01 and more than one intersection between the DEG group and the target gene group. We then constructed a GRN for each DEG group; each GRN was composed of the statistically significant TFs and target genes. Finally, we merged each DEG-specific GRN to construct the final integrated eWAT GRN.

\section{Statistical test}

We performed one-sided two sample $t$-test to compare the weight gain, eWAT weight, total white adipose tissue (WAT) weight, food intake, energy intake, plasma concentration of total cholesterol, and plasma concentration of free fatty acid for mice fed a normal diet (ND), high-fat-diet (HD), high-fat-diet

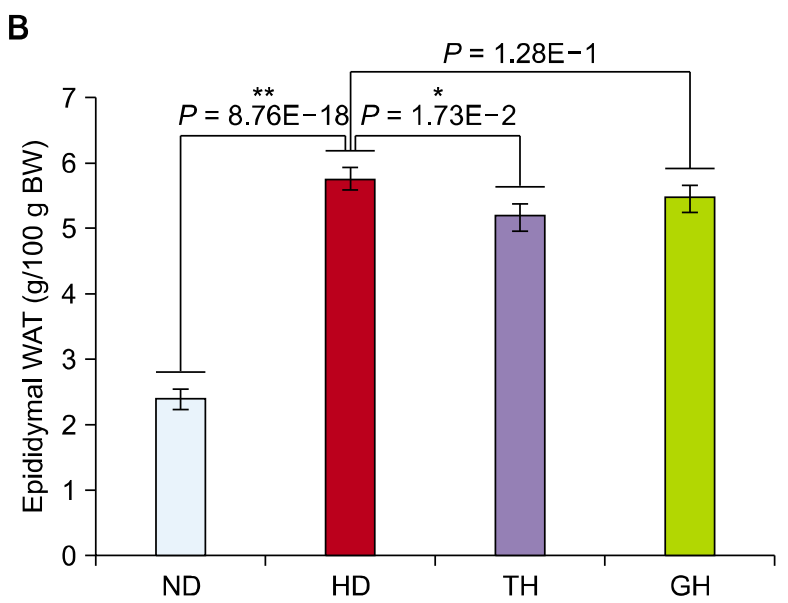

Figure 1. Phenotypic response to a high-fat-diet, Taeumjowitang supplementation, and green tea supplementation: (A) Gain in body weight, (B) weight of eWAT, (C) total WAT weight of ND, HD, TH, and GH mouse groups. The error bar in each graph means standard error. eWAT, epididymal white adipose tissue; WAT, white adipose tissue; ND, mice fed normal diet; HD, mice fed a high-fat-diet; TH, mice fed a high-fat-diet supplemented with Taeumjowitang; GH, mice fed a high-fat-diet supplemented with green tea; BW, body weight. $* P<$ $0.05, * * P<0.001$. 
upplemented with Taeumjowitang (TH), or high-fat-diet supplemented with green tea $(\mathrm{GH})$.

\section{RESULTS}

1. Taeumjowitang and green tea decrease total body weight and relative weights of various adipose tissues in mice fed a high-fat-diet

Characteristic phenotypic changes were observed in $\mathrm{HD}, \mathrm{TH}$, and GH compared to ND. For example, after 12 weeks, the total body weight gain was significantly higher in HD than in ND; however, both Taeumjowitang and green tea attenuated the high-fat-diet induced weight gain (Fig. 1A). The relative weights of eWAT (Fig. 1B) and total WAT (Fig. 1C) were also higher in HD than in ND, and as with total body weight, both Taeumjowitang and green tea reduced the effect of the high-fat-diet; however, the difference was significant in eWAT only for TH but not for GH. These results suggest that both Taeumjowitang and green tea have anti-obesity effects, but Taeumjowitang appears more effective than green tea in eWAT. We also found that the weights of mesenteric WAT, interscapular brown adipose tissue, and visceral fat were significantly higher in HD than in ND; however, both Taeumjowitang and green tea attenuated the weights of various adipose tissues.

Food intake was not significantly different between the HD $(2.67 \pm 0.04 \mathrm{~g} / \mathrm{d}), \mathrm{TH}(2.68 \pm 0.04 \mathrm{~g} / \mathrm{d})$, and GH $(2.62 \pm 0.03 \mathrm{~g} / \mathrm{d})$ groups. However, ND consumed more food $(2.90 \pm 0.04 \mathrm{~g} / \mathrm{d})$ than the three other groups due to the low energy density in the normal diet ( $P=1.03 \mathrm{E}-4,3.43 \mathrm{E}-3,3.56 \mathrm{E}-4$ for HD vs. ND, TH vs. $\mathrm{ND}$, and GH vs. ND, respectively). Energy intake was also not significantly different between $\mathrm{HD}(13.6 \pm 0.17 \mathrm{kcal} / \mathrm{d}), \mathrm{TH}$ (13.3 $\pm 0.2 \mathrm{kcal} / \mathrm{d})$, and GH $(13.4 \pm 0.2 \mathrm{kcal} / \mathrm{d})$. However, energy intake of $\mathrm{ND}(11.0 \pm 0.2 \mathrm{kcal} / \mathrm{d})$ was significantly lower than the three other groups ( $P=6.53 \mathrm{E}-13$, 3.70E-9, 1.13E-9 for HD vs. ND, TH vs. $\mathrm{ND}$, and GH vs. ND, respectively). For plasma lipid concentration, triglyceride concentration was not different among groups ranging from 0.95 to $1.07 \mathrm{mmol} / \mathrm{L}$, but plasma total cholesterol concentration was significantly higher in the $\mathrm{HD}(4.86 \pm 0.15$ $\mathrm{mmol} / \mathrm{L})$ than $\mathrm{ND}(3.41 \pm 0.07 \mathrm{mmol} / \mathrm{L})$ and lower in the TH $(3.92$ $\pm 0.10 \mathrm{mmol} / \mathrm{L})$ and $\mathrm{GH}(3.64 \pm 0.24 \mathrm{mmol} / \mathrm{L})$ than $\mathrm{HD}(P=$ 2.00E-10, 1.43E-4, 9.00E-4 for HD vs. ND, TH vs. HD, and GH vs. $\mathrm{HD}$, respectively). Also, concentration of plasma free fatty acid was significantly lower in the TH $(0.50 \pm 0.02 \mathrm{mmol} / \mathrm{L})$ and GH $(0.51 \pm 0.04 \mathrm{mmol} / \mathrm{L})$ than $\mathrm{HD}(0.60 \pm 0.03 \mathrm{mmol} / \mathrm{L})(P=0.01$ and 0.05 for TH vs. HD and GH vs. HD, respectively).

\section{Taeumjowitang and green tea induce different tissue-specific gene expression profiles}

To identify the genes potentially associated with the anti-obesity effects of Taeumjowitang and green tea, we performed mRNA-seq on eWAT, liver tissue, muscle tissue, and hypothalamus tissue obtained from ND, $\mathrm{HD}, \mathrm{TH}$, and GH. We first identified DEGs by comparing mRNA expression levels between $\mathrm{HD}$ and ND, TH and HD, and GH and HD. Comparing HD and ND, we identified 2,392 DEGs in the eWAT (1,585 up- and 807 down-regulated in HD compared to ND), 1,459 in the liver (1,037 up- and 422 down-regulated in HD compared to ND), 736 in the muscle (442 up- and 294 down-regulated in HD compared to ND), and 343 in the hypothalamus (155 up- and 188 down-regulated in HD compared to ND) (Supplementary Fig. 1). This represents that the eWAT is the most actively responding tissue among the four tissues to diet-induced obesity.

All DEGs identified above were subsequently grouped into six groups for each tissue as follows: UHDTG, UHDT, UHDG, DHUTG, DHUT, and DHUG. An in-depth analysis of these groups revealed tissue-specific transcriptional regulations by Taeumjowitang and/or green tea. The transcriptional alterations associated with a high-fat-diet were more effectively regulated by Taeumjowitang in the eWAT, were similarly regulated by Taeumjowitang and green tea in the liver, and were more effectively regulated by green tea in the muscle and hypothalamus (Fig. 2). The number of UHDT genes was more than sixteen times the number of UHDG genes in the eWAT; likewise, the number of DHUT genes was more than four times the number of DHUG genes (Fig. 2A). Furthermore, the average log ratio of TH : HD was significantly lower than the average log ratio of GH : HD for DEGs up-regulated by a high-fat-diet compared to a normal diet in the eWAT, while the average log ratio of $\mathrm{TH}$ : HD was significantly higher than the average log ratio of $\mathrm{GH}$ : HD for DEGs down-regulated by a high-fat-diet compared to a normal diet (Fig. 2A). In the liver, the number of UHDTG genes was higher than the number of UHDT and UHDG genes, while the number of DHUTG genes was higher than the number of DHUT and DHUG genes (Fig. 2B). Additionally, the average log ratio of $\mathrm{TH}$ : $\mathrm{HD}$ and $\mathrm{GH}$ : $\mathrm{HD}$ was similar for DEGs up-regulated by a high-fat-diet compared to a normal diet in the liver; likewise, the average log ratio of $\mathrm{TH}: \mathrm{HD}$ and $\mathrm{GH}$ : HD was similar for DEGs down-regulated by a high-fat-diet compared to a normal diet (Fig. 2B). In contrast to what we observed in the eWAT, the number of UHDG genes was more than four times the number of UHDT genes and the number of DHUG genes was more than four times the number of DHUT genes in the 
A

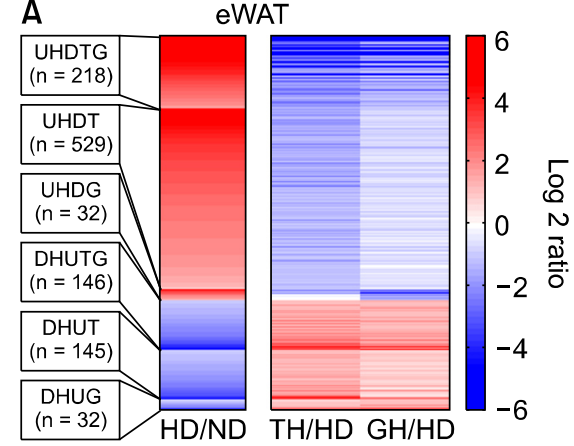

B

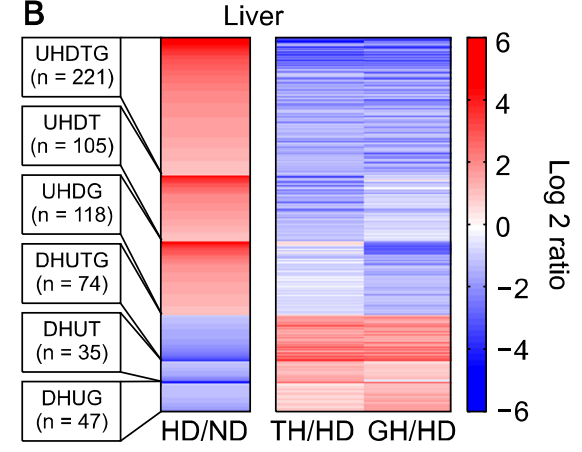

C

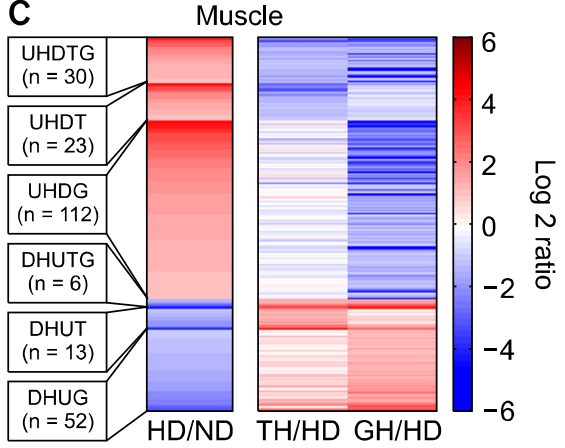

D

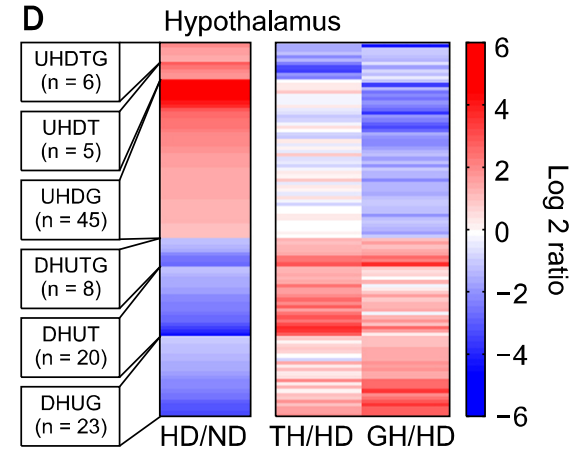

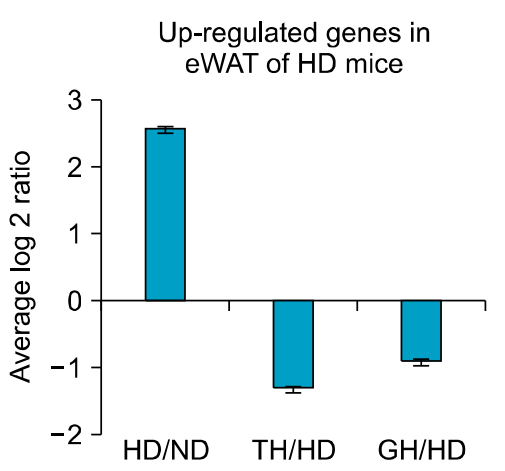
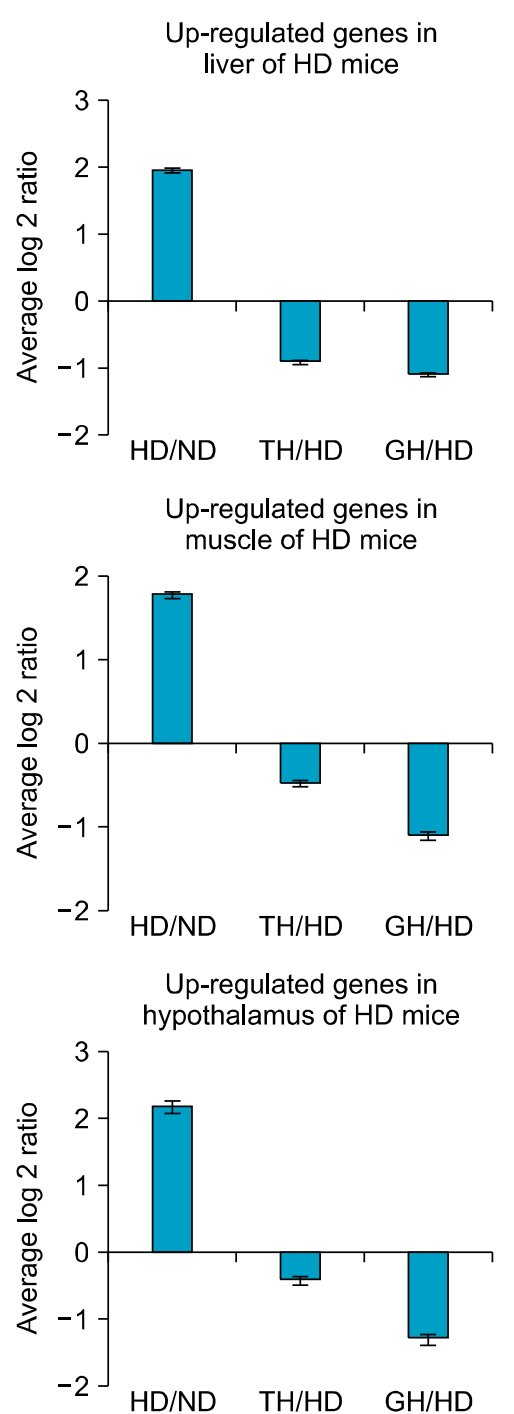
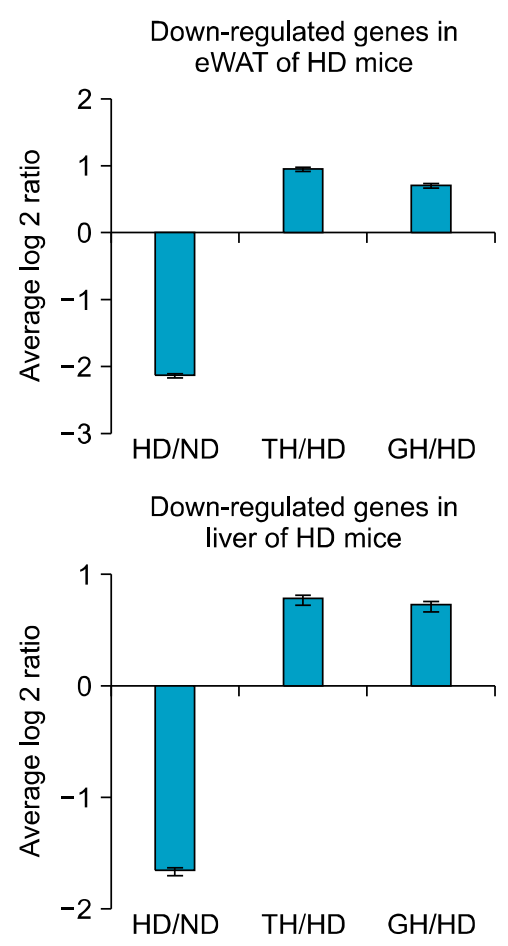

Down-regulated genes in muscle of HD mice

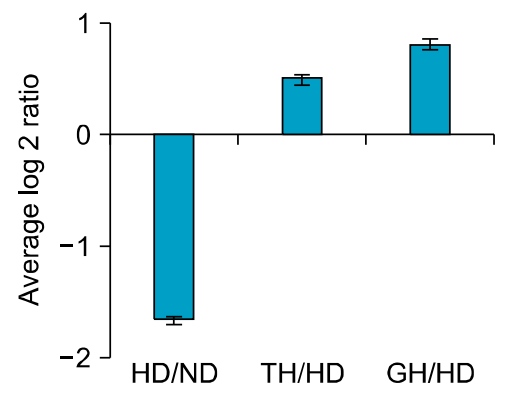

Down-regulated genes in hypothalamus of HD mice

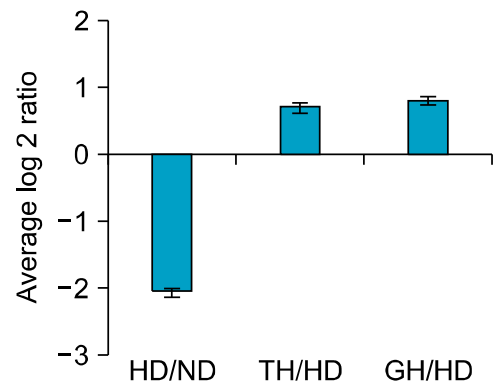

Figure 2. Expression profiles of four tissues in ND, HD, TH, and GH groups. A heat map of six DEG groups (UHDTG, UHDT, UHDG, DHUTG, DHUT, and DHUG), the average log ratio (HD/ND, TH/HD, and GH/HD) of up-regulated DEGs, and the average log ratio (HD/ND, TH/HD, and GH/HD) of down-regulated DEGs, calculated by comparing HD and ND groups in (A) eWAT, (B) liver, (C) muscle, and (D) hypothalamus. ND, mice fed normal diet; HD, mice fed a high-fat-diet; TH, mice fed a high-fat-diet supplemented with Taeumjowitang; GH, mice fed a high-fat-diet supplemented with green tea; UHDTG, up-regulated by HD and down-regulated by Taeumjowitang and green tea; UHDT, up-regulated by HD and down-regulated by Taeumjowitang; UHDG, up-regulated by HD and down-regulated by green tea; DHUTG, down-regulated by HD and up-regulated by Taeumjowitang and green tea; DHUT, down-regulated by HD and up-regulated by Taeumjowitang; DHUG, down-regulated by HD and up-regulated by green tea; eWAT, epididymal white adipose tissue. 

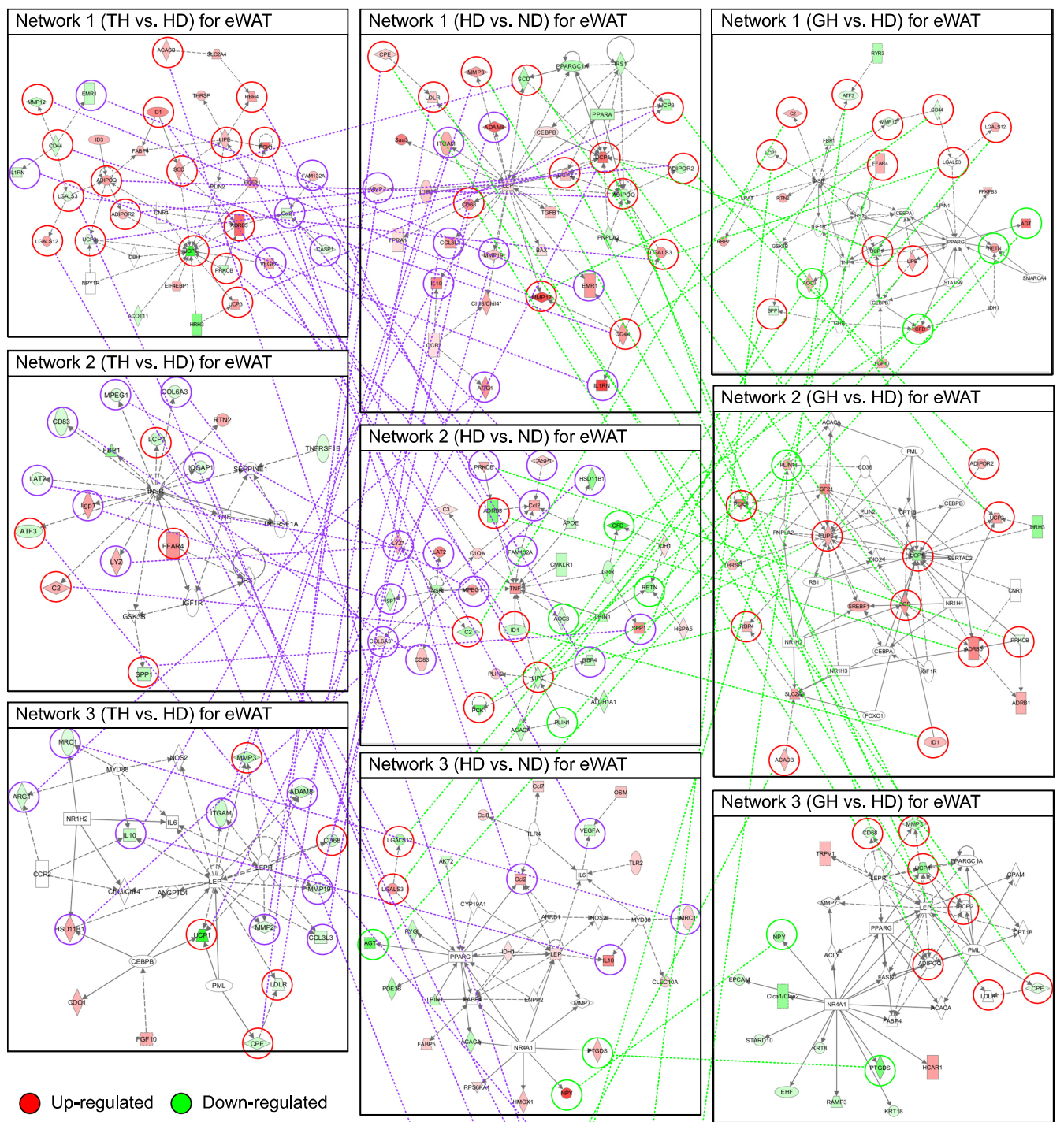

Up-regulated $\bigcirc$ Down-regulated

Commonly regulated by

Taeumjowitang and green tea

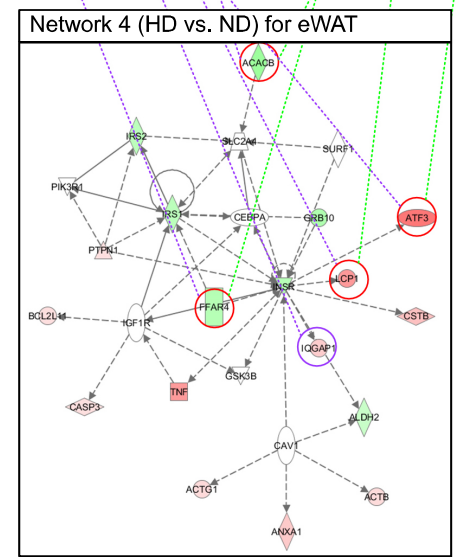

Figure 3. Significant networks altered by a high-fat-diet, Taeumjowitang, and green tea in the eWAT. The significant networks were obtained from software Ingenuity Pathway Analysis. TH, mice fed a high-fat-diet supplemented with Taeumjowitang; HD, mice fed a high-fat-diet; eWAT, epididymal white adipose tissue; ND, mice fed normal diet; GH, mice fed a high-fat-diet supplemented with green tea. 
muscle (Fig. 2C), while in the hypothalamus, the number of UHDG genes was more than nine times the number of UHDT genes (Fig. 2D). The average log ratio of $\mathrm{GH}$ : HD was significantly lower than the average log ratio of $\mathrm{TH}$ : HD for DEGs up-regulated by a high-fat-diet compared to a normal diet, while the average log ratio of $\mathrm{GH}$ : HD was significantly higher than the average log ratio of $\mathrm{TH}$ : HD of down-regulated DEGs in the muscle and hypothalamus (Fig. 2C and 2D). In summary, Taeumjowitang and green tea differentially modulate transcriptomes in a tissue-specific manner even though both have similar weight lowering effects. We also obtained similar results by comparing mRNA expression levels between TH with ND and GH with ND (Supplementary Fig. 2).

\section{Taeumjowitang and green tea differentially regulate molecular networks altered by a high-fat-diet}

To identify the molecular mechanisms underlying the gene expression profiles induced by a high-fat-diet, Taeumjowitang, and green tea, we performed an integrative network analysis using Ingenuity Pathway Analysis (IPA). ${ }^{33}$ Using this tool, we identified four significant networks when comparing HD to ND, three significant networks when comparing TH to HD, three significant networks when comparing GH to HD, four significant networks when comparing $\mathrm{TH}$ to $\mathrm{ND}$, and three significant networks when comparing GH to ND for the eWAT (score $\geq 5$; Supplementary Table 1-5 and Supplementary Fig. 3-7). The networks altered in the HD compared to ND and in the TH compared to HD for the eWAT were commonly associated with 'Nutritional Disease' or 'Metabolic Disease'. In contrast, the networks altered in GH compared to HD were commonly associated with 'Carbohydrate Metabolism'. Networks fitting into this metabolism category were also altered in HD compared to ND. Within the networks altered in HD compared to ND, some nodes ( $A C A C B, A D I P O Q, A D I P O R 2, A D R B 3, A T F 3, C 2, C D 44$, CD68, CPE, FFAR4, ID1, LCP1, LDLR, LGALS3, LGALS12, LIPE, MMP3, MMP12, PCK1, PRKCB, RBP4, SCD, SPP1, UCP1, UCP2, and $U C P 3)$ were commonly regulated by Taeumjowitang and green tea, some nodes (ADAM8, ARG1, CCL3L3, IL10, IL1RN, ITGAM, MMP2, MMP19, CASP1, CCl2, CD83, COL6A3, FAM132A, HSD11B1, ligp1, LAT2, LYZ, MPEG1, MRC1, and VEGFA) were regulated only by Taeumjowitang, whereas other nodes (AOC3, CFD, PLIN1, RETN, AGT, NPY, and PTGDS) were regulated only by green tea (Fig. 3). We also found that a canonical pathway associated with 'leukocyte extravasation signaling' was activated by a high-fat-diet but was attenuated by Taeumjowitang, which means that most of the proteins in this pathway were down-regulated by Taeumjowitang (Fig. 4 and Supplementary Table 6-10). In contrast, few proteins in the leukocyte extravasation signaling pathway were down-regulated by green tea.

IPA based on the liver transcriptome identified fourteen significant networks when comparing HD to ND, eight significant networks when comparing $\mathrm{TH}$ to $\mathrm{HD}$, eight significant networks when comparing GH to HD, seven significant networks when comparing $\mathrm{TH}$ to $\mathrm{ND}$, and nine significant networks when comparing GH to ND (score $\geq 5$; Supplementary Table 11-15 and Supplementary Fig. 8-12). The significant networks altered in the HDvs. ND, those altered in the TH vs. HD, and those altered in the GH vs. HD for the liver were commonly associated with 'lipid metabolism', 'molecular transport', and 'small molecule biochemistry'. This represents that Taeumjowitang and green tea may commonly regulate lipid metabolic processes in the liver. We also found that a canonical pathway associated with 'hepatic fibrosis/hepatic stellate cell activation' was commonly regulated by a high-fat-diet, Taeumjowitang and green tea in the liver (Supplementary Table 16-20).

For the muscle transcriptome, we identified one significant network when comparing $\mathrm{HD}$ to ND, one significant network when comparing $\mathrm{TH}$ to $\mathrm{HD}$, one significant network when comparing GH to HD, one significant network when comparing TH to ND, and one significant network when comparing GH to ND (score $\geq 5$; Supplementary Table 21-25 and Supplementary Fig. 13). The networks altered in the HD compared to ND or in the GH compared to HD for the muscle were commonly associated with 'lipid metabolism' or 'molecular transport'. In contrast, the network altered in GH compared to HD was associated with 'organismal functions', 'hematological disease', and 'metabolic disease'. This represents that green tea may more effectively regulate lipid metabolic processes than Taeumjowitang in the muscle. In the case of hypothalamus, we could not find any significant networks in IPA based on the DEGs in HD, TH, and GH.

4. Taeumjowitang and green tea regulate different modules in the integrated epididymal white adipose tissue gene regulatory network

To identify significant regulators of the eWAT transcriptome response to a high-fat-diet, Taeumjowitang, or green tea, we constructed the 'integrated eWAT GRN for obesity treatment' based on the six DEG groups described above (UHDTG, UHDT, UHDG, DHUTG, DHUT, and DHUG) (Fig. 5). Due to the size and complexity of the GRN (427 nodes and 2,455 links), we modularized the network using the Newman algorithm ${ }^{34}$ in the Cytoscape plugin clusterMaker ${ }^{35}$ and subsequently identified five modules. To characterize the functional annotations of the genes 


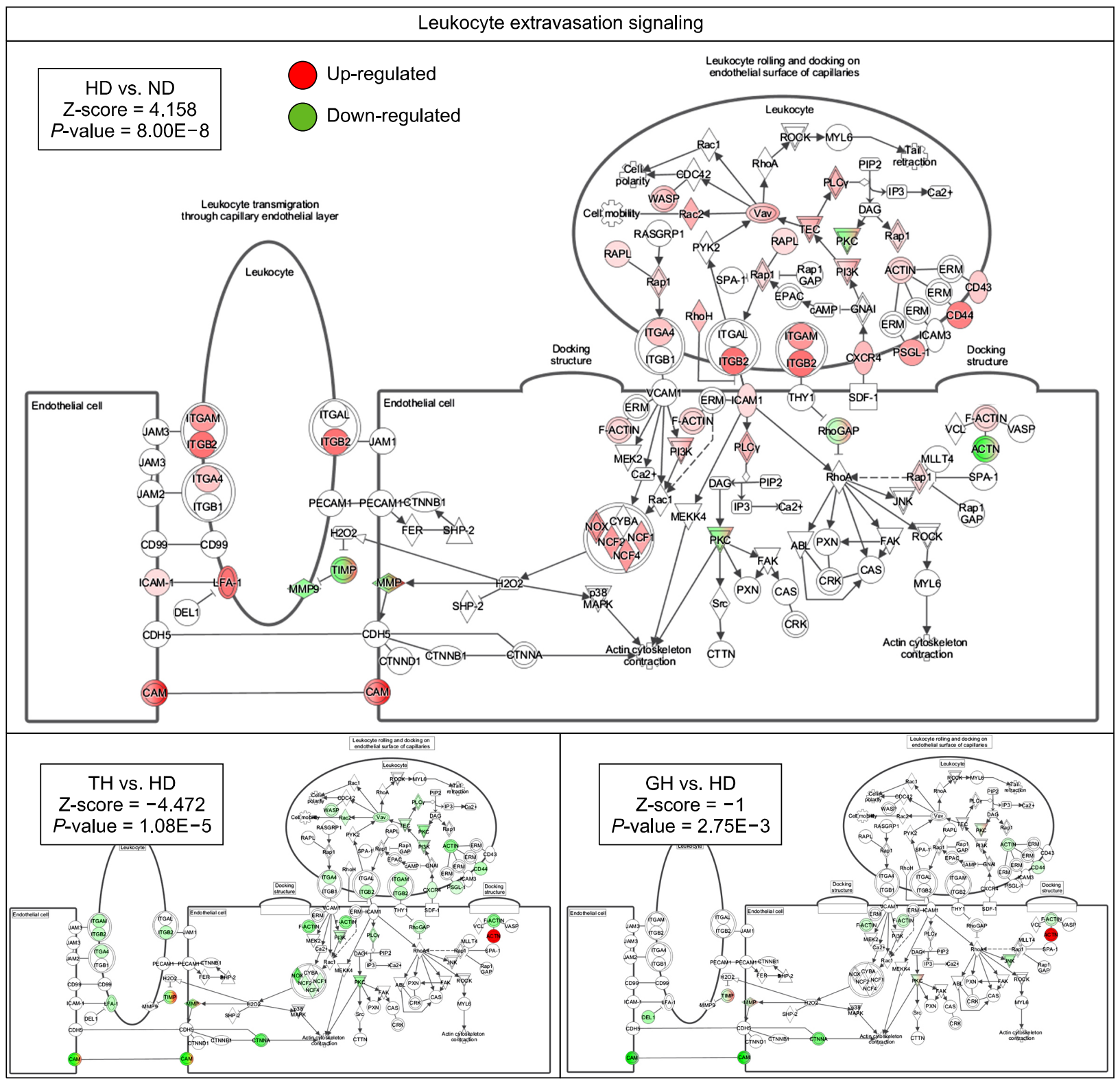

Figure 4. Leukocyte extravasation signaling pathway altered by a high-fat-diet, Taeumjowitang, and green tea in the epididymal white adipose tissue. This pathway were obtained from software Ingenuity Pathway Analysis. HD, mice fed a high-fat-diet; ND, mice fed normal diet; TH, mice fed a high-fat-diet supplemented with Taeumjowitang; GH, mice fed a high-fat-diet supplemented with green tea.

present in each module, we used a functional annotation tool DAVID to perform a gene set enrichment analysis. ${ }^{30}$ Using this approach, we identified four well-characterized modules. Module 1, which was mainly comprised of UHDTG and UHDT genes, was associated with the GO terms 'immune response' and 'defense response' (Supplementary Table 26). Module 2, which was mostly comprised of UHDT genes, was associated with the GO terms 'intracellular signaling cascade', 'lymphocyte activation', 'leukocyte activation', and 'regulation of cytokine production' (Supplementary Table 27). Module 3, which was mostly comprised of
UHDG and DHUTG genes, was associated with the GO terms 'chemical homeostasis' and 'gluconeogenesis' (Supplementary Table 28). Module 4, which was comprised of UHDT genes, was associated with the GO term 'cell cycle' (Supplementary Table 29). This GRN analysis revealed that Taeumjowitang and green tea regulate different functional modules of the integrated GRN.

\section{DISCUSSION}

Obesity is associated to morbidities of cardiovascular disease, 


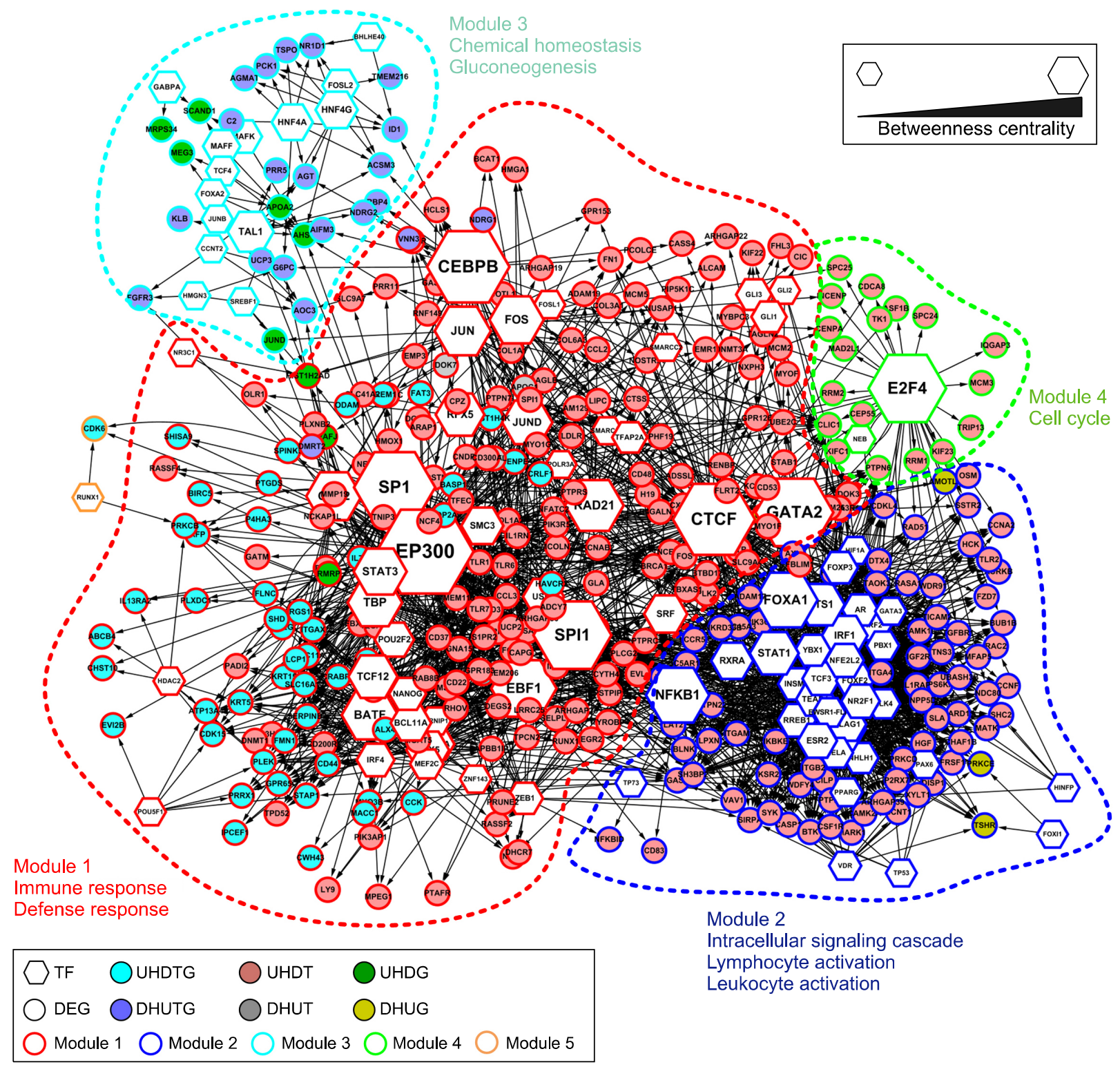

Figure 5. An integrated epididymal white adipose tissue gene regulatory network. Hexagon nodes, and circle nodes denoting TFs and DEGs, respectively. Bright blue, bright red, green, blue, grey, and gold nodes denote UHDTG, UHDT, UHDG, DHUTG, DHUT, and DHUG, respectively. The nodes with red, blue, bright blue, bright green, and orange border line nodes denote TFs or DEGs in Modules 1 to 5, respectively. TF, transcription factor; DEG, differentially expressed gene; UHDTG, up-regulated by mice fed a high-fat-diet (HD) and down-regulated by Taeumjowitang and green tea; UHDT, up-regulated by HD and down-regulated by Taeumjowitang; UHDG, up-regulated by HD and down-regulated by green tea; DHUTG, down-regulated by HD and up-regulated by Taeumjowitang and green tea; DHUT, down-regulated by HD and up-regulated by Taeumjowitang; DHUG, down-regulated by HD and up-regulated by green tea.

type 2 diabetes mellitus, and cancer. Obesity increases the risk for development of various cancers including lung, breast, liver, kidney, gastric, and pancreatic cancer. Controlling obesity is one of the important factors for cancer prevention.

Here, we describe for the first time a systematic analysis of transcriptome data for four tissues collected from C57BL/6J mice fed a normal diet, a high-fat-diet, a high-fat-diet supplemented with Taeumjowitang, or a high-fat-diet supplemented with green tea. We found that tissue-specific transcriptomic modulations were differentially induced by the two supplements, even though both similarly reduced body mass. While both Taeumjowitang and green tea induced gene expression alterations in all four tissues analyzed (eWAT, liver, muscle, and hypothalamus), differential effects were observed. While Taeumjowitang was a more effective regulator of the eWAT transcriptome altered by a high-fat-diet, green tea was a more effective regulator of the 
muscle and hypothalamus transcriptomes altered by a high-fat-diet, and both Taeumjowitang and green tea similarly regulated liver-specific transcriptome altered by a high-fat-diet. Within the eWAT, Taeumjowitang appeared to be a more effective regulator of the pathological alterations than green tea, and green tea appeared to be a more effective regulator of carbohydrate metabolism. The differential tissue-specific transcriptomic modulations induced by Taeumjowitang and green tea suggest that the underlying mechanisms behind the anti-obesity effects of these two traditional medicines are likely quite different.

eWAT actively contributes not only to whole body homeostasis but also to immunity and inflammation by secreting various adipokines. ${ }^{36}$ Our functional network analyses identified a GRN module and a pathway associated with 'immune cell activation' that responded to Taeumjowitang but not to green tea in the eWAT, indicating that Taeumjowitang is better able to inhibit inflammation in the eWAT. Among the eight components comprising Taeumjowitang, ${ }^{12}$ Radix Platycodi and Radix Liriope platyphylla contain high levels of saponins, which have been reported to have anti-inflammatory effects. ${ }^{20,37}$ Platycodin D, isolated from Radix Platycodi, induces anti-inflammatory effects by inhibiting the activity of NF- $\mathrm{KB}$, a major inflammation-associated TF, in macrophages. ${ }^{38}$ Platycodin $\mathrm{D}$ also inhibits the expression of the NF-kB targets, inducible nitric oxide synthase and COX-2. ${ }^{38}$ Similarly, prosapogenin III, isolated from Radix Liriope platyphylla, also inhibits the NF- $\mathrm{KB}$ pathway. ${ }^{37}$ In contrast, catechins in green tea have weak anti-inflammatory effects $^{39}$. These studies support our observations, and we therefore hypothesize that the anti-inflammatory gene expression alterations induced by Taeumjowitang may be due to the anti-inflammatory properties of Radix Platycodi and Radix Liriope platyphylla.

A canonical pathway associated with 'Heparan sulfate biosynthesis' was commonly altered by Taeumjowitang and green tea in the eWAT (Supplementary Table 7 and 8). Heparan sulfate proteogycans, which reside on the plasma membrane of all animal cells, are known to have essential roles in the integration of physical and chemical activities to maintain systemic homeostasis. ${ }^{40}$ Our findings on the common alteration of the heparan sulfate biosynthesis pathway by Taeumjowitang and green tea indicate that the two traditional oriental medicines may have common effects on the homeostatic maintenance against diet-induced physiological perturbations.

We additionally constructed a functional gene network to identify significant regulators of the gene expression patterns observed in eWAT. We identified four functional modules, two of which (Modules 2 and 4) were comprised of DEGs that were down-regulated by Taeumjowitang but not by green tea. Down-regulated DEGs were categorized into GO categories 'intracellular signaling cascade', 'lymphocyte activation', 'leukocyte activation', and 'cell cycle'. Module 4 also included NF-кB, further supporting the anti-inflammatory effects that seem to be attributed to Taeumjowitang. Module 3 was composed of DEGs categorized into GO categories 'chemical homeostasis' and 'gluconeogenesis'. These DEGs were down-regulated only by green tea. Together, these results suggest that Taeumjowitang has more powerful anti-inflammatory effects than green tea, but green tea may more effectively regulate glucose metabolism, and that these differential effects may be due to the modulation of the eWAT transcriptome.

Several studies have revealed that (-)-epigallocatechin-3-gallate (EGCG) in green tea increases lipid oxidation and the expressions of genes related to the lipid oxidation in murine muscle. ${ }^{41,42}$ Lipid oxidation in skeletal muscle is closely associated with insulin resistance, ${ }^{43}$ an obesity-associated complication. To confirm the relationship between insulin resistance and green tea in our mRNA-seq data, we investigated the DEG group UHDG in the muscle transcriptome. We found that in muscle tissue, green tea (but not Taeumjowitang) down-regulated several genes associated with lipid metabolism (Acaa1b, Apoa4, Apoa5, Apoc2, and Apoc4) that were up-regulated by a high-fat-diet (Supplementary Fig. 14). Furthermore, only green tea could down-regulate the insulin transactivator, v-maf musculoaponeurotic fibrosarcoma oncogene family, and protein A (Mafa), which was also up-regulated by a high-fat-diet (Supplementary Fig. 14). These data suggest that insulin resistance in the muscle may be more effectively regulated by green tea than Taeumjowitang.

Biological targeting organisms have evolved to suggest that their innate molecular networks have distinct structures enriched in feedback loops and cross-regulation between different pathways, which ensures network robustness against various perturbations. ${ }^{44,45}$ Due to this robust nature, resistance to several targeted drugs for the treatment of obesity has been reported. ${ }^{45}$ To overcome drug resistance, agent comprised of multiple components and several molecular pathways have emerged as new alternatives against using single agent to target a single mechanism. ${ }^{5}$ We observed differential tissue-specific and pathway-specific anti-obesity effects of Taeumjowitang and green tea, suggesting that these two traditional medicines may represent an effective and natural combination therapy for obesity with a low risk for resistance. Future studies treating animal models with both treatments simultaneously will be 
essential to prove this hypothesis, and if proven effective and this treatment strategy could revolutionize the treatment of obesity.

\section{ACKNOWLEDGMENTS}

This work was supported by a grant of the Korea Health Technology R\&D Project through the Korea Health Industry Development Institute (KHIDI), funded by the Ministry of Health \& Welfare, Republic of Korea (grant number: HI14C2640). We thank to Jin Muk Kang, Bona Lee, Kyung-Min Yang, Yuna Park, Jihee Lee, Kyoungwha Pang, Bora Park for their valuable comments on this article.

\section{CONFLICTS OF INTEREST}

No potential conflicts of interest were disclosed.

\section{SUPPLEMENTARY MATERIALS}

Supplementary materials can be found via https://doi.org/ 10.15430/JCP.2017.22.3.147.

\section{REFERENCES}

1. Eckel RH, Grundy SM, Zimmet PZ. The metabolic syndrome. Lancet 2005:365:1415-28.

2. Rocha VZ, Libby P. Obesity, inflammation, and atherosclerosis. Nat Rev Cardiol 2009:6:399-409.

3. Shoelson SE, Herrero L, Naaz A. Obesity, inflammation, and insulin resistance. Gastroenterology 2007;132:2169-80.

4. Sun B, Karin M. Obesity, inflammation, and liver cancer. J Hepatol 2012;56:704-13.

5. Kim HU, Ryu JY, Lee JO, Lee SY. A systems approach to traditional oriental medicine. Nat Biotechnol 2015:33:264-8.

6. Kim J, Park SM, Cho KH. Discovery of a kernel for controlling biomolecular regulatory networks. Sci Rep 2013;3:2223.

7. Dulloo AG, Seydoux J, Girardier L, Chantre P, Vandermander J. Green tea and thermogenesis: interactions between catechin-polyphenols, caffeine and sympathetic activity. Int J Obes Relat Metab Disord 2000:24:252-8.

8. Zheng G, Sayama K, Okubo T, Juneja LR, Oguni I. Anti-obesity effects of three major components of green tea, catechins, caffeine and theanine, in mice. In Vivo 2004; 18:55-62.

9. Dulloo AG, Duret C, Rohrer D, Girardier L, Mensi N, Fathi M, et al. Efficacy of a green tea extract rich in catechin polyphenols and caffeine in increasing 24-h energy expenditure and fat oxidation in humans. Am J Clin Nutr 1999; 70:1040-5.

10. Yan J, Zhao Y, Suo S, Liu Y, Zhao B. Green tea catechins ameliorate adipose insulin resistance by improving oxidative stress. Free Radic Biol Med 2012;52:1648-57.

11. Ahmad RS, Butt MS, Sultan MT, Mushtaq Z, Ahmad S, Dewanjee $S$, et al. Preventive role of green tea catechins from obesity and related disorders especially hypercholesterolemia and hypergly- cemia. J Transl Med 2015:13:79.

12. Kim SW, Park TJ, Choi JH, Aseer KR, Choi JY, Kim YJ, et al. Differential protein expression in white adipose tissue from obesity-prone and obesity-resistant mice in response to high fat diet and anti-obesity herbal medicines. Cell Physiol Biochem 2015; 35:1482-98.

13. Yoo JH, Lee EJ, Kwak CK, Sohn EH, Koh BH, Song IB, et al. Clinical trial of herbal formula on weight loss in obese Korean children. Am J Chin Med 2005:33:713-22.

14. Park S, Nahmkoong W, Cheon C, Park JS, Jang BH, Shin Y, et al. Efficacy and safety of taeeumjowi-tang in obese Korean adults: a double-blind, randomized, and placebo-controlled pilot trial. Evid Based Complement Alternat Med 2013;2013:498935.

15. Lee H, Bae S, Kim YS, Yoon Y. WNT/ $\beta$-catenin pathway mediates the anti-adipogenic effect of platycodin $\mathrm{D}$, a natural compound found in Platycodon grandiflorum. Life Sci 2011;89:388-94.

16. Lee H, Kang R, Kim YS, Chung SI, Yoon Y. Platycodin D inhibits adipogenesis of 3T3-L1 cells by modulating Kruppel-like factor 2 and peroxisome proliferator-activated receptor gamma. Phytother Res 2010;24 Suppl 2:S161-7.

17. Lee EJ, Kang M, Kim YS. Platycodin D inhibits lipogenesis through AMPK $\alpha$-PPAR $\gamma 2$ in 3T3-L1 cells and modulates fat accumulation in obese mice. Planta Med 2012;78:1536-42.

18. Hwang YP, Choi JH, Kim HG, Lee HS, Chung YC, Jeong HG. Saponins from Platycodon grandiflorum inhibit hepatic lipogenesis through induction of SIRT1 and activation of AMP-activated protein kinase in high-glucose-induced HepG2 cells. Food Chem 2013;140:115-23.

19. Hwang YP, Choi JH, Kim HG, Khanal T, Song GY, Nam MS, et al. Saponins, especially platycodin D, from Platycodon grandiflorum modulate hepatic lipogenesis in high-fat diet-fed rats and high glucose-exposed HepG2 cells. Toxicol Appl Pharmacol 2013;267: $174-83$.

20. Chung JW, Noh EJ, Zhao HL, Sim JS, Ha YW, Shin EM, et al. Anti-inflammatory activity of prosapogenin methyl ester of platycodin D via nuclear factor-kappaB pathway inhibition. Biol Pharm Bull 2008;31:2114-20.

21. Li T, Xu WS, Wu GS, Chen XP, Wang YT, Lu JJ. Platycodin D induces apoptosis, and inhibits adhesion, migration and invasion in HepG2 hepatocellular carcinoma cells. Asian Pac J Cancer Prev 2014:15:1745-9.

22. Lee H, Kang R, Yoon Y. SH21B, an anti-obesity herbal composition, inhibits fat accumulation in 3T3-L1 adipocytes and high fat diet-induced obese mice through the modulation of the adipogenesis pathway. J Ethnopharmacol 2010;127:709-17.

23. Matsui N, Ito R, Nishimura E, Yoshikawa M, Kato M, Kamei M, et al. Ingested cocoa can prevent high-fat diet-induced obesity by regulating the expression of genes for fatty acid metabolism. Nutrition 2005:21:594-601.

24. Aoki F, Honda S, Kishida H, Kitano M, Arai N, Tanaka H, et al. Suppression by licorice flavonoids of abdominal fat accumulation and body weight gain in high-fat diet-induced obese C57BL/6J mice. Biosci Biotechnol Biochem 2007;71:206-14.

25. Reagan-Shaw S, Nihal M, Ahmad N. Dose translation from animal to human studies revisited. FASEB J 2008;22:659-61.

26. Dobin A, Davis CA, Schlesinger F, Drenkow J, Zaleski C, Jha S, et al. STAR: ultrafast universal RNA-seq aligner. Bioinformatics 2013:29:15-21.

27. Trapnell C, Williams BA, Pertea G, Mortazavi A, Kwan G, van 
Baren MJ, et al. Transcript assembly and quantification by RNA-Seq reveals unannotated transcripts and isoform switching during cell differentiation. Nat Biotechnol 2010;28:511-5.

28. Anders S, Pyl PT, Huber W. HTSeq: a Python framework to work with high-throughput sequencing data. Bioinformatics 2015;31: 166-9.

29. Sun J, Nishiyama T, Shimizu K, Kadota K. TCC: an R package for comparing tag count data with robust normalization strategies. BMC Bioinformatics 2013;14:219.

30. Huang da W, Sherman BT, Lempicki RA. Systematic and integrative analysis of large gene lists using DAVID bioinformatics resources. Nat Protoc 2009:4:44-57.

31. Gerstein MB, Kundaje A, Hariharan M, Landt SG, Yan KK, Cheng C, et al. Architecture of the human regulatory network derived from ENCODE data. Nature 2012;489:91-100.

32. Fazekas D, Koltai M, Türei D, Módos D, Pálfy M, Dúl Z, et al. SignaLink 2: a signaling pathway resource with multi-layered regulatory networks. BMC Syst Biol 2013;7:7.

33. Krämer A, Green J, Pollard J Jr, Tugendreich S. Causal analysis approaches in ingenuity pathway analysis. Bioinformatics 2014:30: 523-30.

34. Newman ME. Modularity and community structure in networks. Proc Natl Acad Sci U S A 2006;103:8577-82.

35. Morris JH, Apeltsin L, Newman AM, Baumbach J, Wittkop T, Su $\mathrm{G}$, et al. clusterMaker: a multi-algorithm clustering plugin for Cytoscape. BMC Bioinformatics 2011;12:436.

36. Lago F, Dieguez C, Gómez-Reino J, Gualillo O. Adipokines as emerging mediators of immune response and inflammation. Nat Clin Pract Rheumatol 2007;3:716-24.

37. Han Y, Jung HW, Lee DH, Kwon SY, Son KH, Park YK. Anti-inflammatory effects of prosapogenin III from the dried roots of
Liriope platyphylla in LPS-stimulated RAW264.7 cells. J Asian Nat Prod Res 2013;15:1038-49.

38. Ahn KS, Noh EJ, Zhao HL, Jung SH, Kang SS, Kim YS. Inhibition of inducible nitric oxide synthase and cyclooxygenase II by Platycodon grandiflorum saponins via suppression of nuclear factor-kappaB activation in RAW 264.7 cells. Life Sci 2005;76:2315-28.

39. Liang YC, Huang YT, Tsai SH, Lin-Shiau SY, Chen CF, Lin JK. Suppression of inducible cyclooxygenase and inducible nitric oxide synthase by apigenin and related flavonoids in mouse macrophages. Carcinogenesis 1999:20:1945-52.

40. Gordts PL, Esko JD. Heparan sulfate proteoglycans fine-tune macrophage inflammation via IFN- $\beta$. Cytokine 2015;72:118-9.

41. Murase T, Haramizu S, Shimotoyodome A, Nagasawa A, Tokimitsu I. Green tea extract improves endurance capacity and increases muscle lipid oxidation in mice. Am J Physiol Regul Integr Comp Physiol 2005;288:R708-15.

42. Sae-Tan S, Grove KA, Kennett MJ, Lambert JD. (-)-Epigallocatechin-3-gallate increases the expression of genes related to fat oxidation in the skeletal muscle of high fat-fed mice. Food Funct 2011:2:111-6.

43. Kelley DE, Goodpaster B, Wing RR, Simoneau JA. Skeletal muscle fatty acid metabolism in association with insulin resistance, obesity, and weight loss. Am J Physiol 1999:277:E1130-41.

44. Kim J, Vandamme D, Kim JR, Munoz AG, Kolch W, Cho KH. Robustness and evolvability of the human signaling network. PLoS Comput Biol 2014;10:e1003763.

45. Won JK, Yang HW, Shin SY, Lee JH, Heo WD, Cho KH. The crossregulation between ERK and PI3K signaling pathways determines the tumoricidal efficacy of MEK inhibitor. J Mol Cell Biol 2012;4:153-63. 